
associated with the formation of the myelin sheath of neurons in the prefrontal cortex of 15-day-old male mice

\author{
Anastasia S. Shulyupova \\ Laboratory of Gene Expression Regulation \\ Institute of Cytology and Genetics SB RAS \\ Novosibirsk, Russia \\ shulyupova@bionet.nsc.ru \\ Vasiliy V. Reshetnikov \\ Laboratory of Gene Expression Regulation \\ Institute of Cytology and Genetics SB RAS \\ Novosibirsk, Russia \\ vasiliyreshetnikov@bionet.nsc.ru
}

\author{
Arina A. Smelova \\ Epigenetics laboratory \\ National Research Novosibirsk State University \\ Novosibirsk, Russia \\ smelovarina@gmail.com \\ Natalya P. Bondar \\ Laboratory of Gene Expression Regulation \\ Institute of Cytology and Genetics SB RAS \\ Novosibirsk, Russia \\ nbondar@bionet.nsc.ru
}

\begin{abstract}
The early postnatal period is critical for the development of the central nervous system and can predetermine subsequent behavioral patterns, so stress at this age can lead to long-term consequences in adulthood. In our work, we investigated effects early life stress on myelination. We assessed the expression of the genes associated with the myelin sneath (myelin basic protein - Mbp, myelin-associated oligodendrocyte basic protein - Mobp, proteolipid protein 1 PIp1, proteolipid in compact myelin - Mal, myelin oligodendrocyte glycoprotein - Mog, choline-specific glycerophosphodiester-phosphodiesterase - Enpp6, UDPglucuronosyltransferase - Ugt8a) in frontal cortex of mice. The greatest difference in the level of expression was found between groups with a maternal deprivation and a maternal separation from mothers: significant changes were shown for the Enpp6, Mal, Ugt8a genes and one of the Mobp gene transcripts. This may indicate a different extent of exposure to stressors. We can conclude that the stress in the early postnatal period affects the myelination process and can lead to impaired transmission of nerve impulses.
\end{abstract}

Key words - effects of stress; brain; myelination; mice; pups

\section{Motivation and Aim}

The early postnatal period is critical for the development of the central nervous system and can predetermine subsequent behavioral patterns, so stress at this age can lead to long-term consequences in adulthood. Thus, on animal models has been shown that a maternal separation (3 hours) pups from their mothers leads to interruption in the continuity of interaction with the mother and causes severe stress in the pups. Upon reaching adulthood in such animals, an increase in sensitivity to stress, increased anxiety and a number of cognitive disorders (deterioration of spatial memory, ability to recognize objects, etc.) are observed. A previous study of a transcriptome of the prefrontal cortex of 15 day old pups showed that this stress leads to a change in the expression of a large number of genes. Among them, a cluster of genes associated with the formation of the myelin sheath of axons was isolated. The aim of this work was to study the change in the expression of Mbp, Mobp, Plp1, Mal, Mog, Enpp6, Ugt8a genes associated with the formation of the myelin sheath of neurons in the prefrontal cortex of 15 day old male mice under the influence of early postnatal stress. In addition, the effect of stress on physiological (animal weight, adrenal gland weight) and biochemical (blood corticosterone level) parameters was evaluated.

\section{Materials and Methods}

\section{Materials}

To obtain experimental offspring, female mice (28 animals) were planted with males in the ratio of 3 females to 1 male. There were 6 to 9 pups in the litter. Two types of early postnatal stress, conducted from PND2 to PND14, were used in the study: maternal separation of the pups from their mothers ( 3 hours a day daily, MS) and a maternal deprivation (for a day, MD). The control group did not separate from mothers. The pups were separated daily from 13 to 16 hours. On the 15th day of life, animals were killed by decapitation, blood was collected, the prefrontal cortex was isolated and frozen in liquid nitrogen. The adrenal glands were isolated and weighed. RNA was subsequently isolated from frozen tissue samples, and complementary DNA was synthesized based on it.

\section{Methods}

A TaqMan probe-based real-time PCR to evaluate gene expression was used. We evaluated the expression of the main myelin sheath genes: Mbp - encodes a protein that is the main component of the myelin sheath of oligodendrocytes and Schwann cells in the nervous system, Mobp - encodes the main oligodendrocyte protein associated with myelin (evaluated using two pairs of primers covering all known gene transcripts), Plp1 - encodes a proteolipid protein, which plays an important role in the formation and maintenance of the multilayer structure of myelin, Mal - encodes a proteolipid involved in myelin biogenesis. In the studied groups of animals, body weight and organ index of the adrenal glands were evaluated. The organ index was calculated by the formula: OI $=$ adrenal gland weight $/$ body weight $* 100 \%$. The level of corticosterone was measured in the blood serum of pups on PND 15 by ELISA. 


\section{Results}

The effect of stress on the physiological characteristics of pups

The stress factor did not significantly affect the body weight of the pups. However, stress during the first two weeks of life had an effect on the relative weight of the adrenal glands: in the MD group, in comparison with the MS group, the organ adrenal gland index was significantly reduced in pups. The level of corticosterone did not differ significantly between different experimental groups, however, in mice from the MS group there was a tendency $(\mathrm{p}<0.073)$ to lower levels compared with the control group.

The effect of stress on gene expression in the frontal cortex

Analysis of gene expression in the frontal cortex showed a significant increase $(\mathrm{p}=0.038)$ in the expression level of the Mal gene in the MD group compared to the MS group and a tendency to increase $(\mathrm{p}=0.053)$ compared to the control group. The Mobp gene is characterized by the presence of many alternative transcripts. Since the gene structure does not allow choice of primers covering all isoforms, we estimated the expression level using two pairs of primers. One pair covers the transcripts Mobp$201,202,204,206,207$, the second pair is specific only for the transcript ENSMUST00000174193.7 (Mobp-204). For the first pair of primers of the Mobp gene, the analysis of PCR-RT data did not reveal significant differences between the experimental groups ( $p>0.05)$, however, the change in the expression of its transcript ENSMUST00000174193.7 was significantly reduced $(\mathrm{p}=0.03)$ in the MS group compared with the MD group. For the Ugt8a gene, a tendency towards an increase in the expression level $(\mathrm{p}=0.07)$ in the MD group as compared with the control was revealed, and a significant increase in the expression level of the Ugt8a gene in the MD group compared to the MS group $(\mathrm{p}=0.04)$. Analysis of Enpp6 gene expression showed that in the MD group the expression level is higher compared to the control $(\mathrm{p}=$ 0.02 ) and the MS group ( $p=0.011)$. Analysis of the expression of the Mbp, Plp1, and Mog genes did not show significant differences in the expression level between the groups.

The greatest difference in the level of expression was found between groups with a maternal deprivation and a maternal separation from mothers: significant changes were shown for the Enpp6, Mal, Ugt8a genes and one of the Mobp gene transcripts. This may indicate a different extent of exposure to stressors. We can conclude that the experience of early stress affects the myelination process and can lead to impaired transmission of nerve impulses.

\section{ACKNOWLEDGMENT}

Supported by the Russian Science Foundation (16-15-10131).

\section{REFERENCES}

[1] Bondar, N. P., Lepeshko, A. A., \& Reshetnikov, V. V. (2018). Effects of early-life stress on social and anxiety-like behaviors in adult mice: sex-specific effects. Behavioural neurology, 2018(2), 1-13.

[2] Mehta, M., \& Schmauss, C. (2011). Strain-specific cognitive deficits in adult mice exposed to early life stress. Behavioral neuroscience, 125(1), 29.

[3] Sakhai, S. A., Saxton, K., \& Francis, D. D. (2016). The influence of early maternal care on perceptual attentional set shifting and stress reactivity in adult rats. Developmental psychobiology, 58(1), 39-51. 